\title{
MICROSURGICAL TECHNIQUES USING HUMAN PLACENTA
}

\author{
Flávio Ramalho Romero', Sérgio Tadeu Fernandes ', Feres Chaddad-Neto², \\ Javier Gonzalez Ramos', Jose Maria de Campos', Evandro de Oliveira ${ }^{3}$
}

Training in microsurgical techniques is the first step to allow procedures in the central nervous system. One of them is the dissection of human placenta. Others include mannequin head', virtual reality environment ${ }^{2}$, animal and cadaver dissections ${ }^{3}$. The human placenta is a maternalfetal structure constituted of a bigger fetal portion and a smaller maternal portion. The fetal membranes and the placenta are responsible for protection, nutrition, breath, excretion and hormone production ${ }^{4}$. The placenta usually shows an ovoid shape with a $16-20 \mathrm{~cm}$ diameter and a 2-3 cm thickness ${ }^{4}$. It weighs between 500 and $600 \mathrm{gr}^{4}$. The umbilical cord is usually composed of a vein and two arteries. The fetal surface has a fetal corion - amniotic membrane which is very similar to the cerebral arachnoid and a rich vascular component with 1-6 mm diameter vessels $\mathrm{s}^{4}$. The anterior cerebral artery has $1-3 \mathrm{~mm}$ diameter and the medium cerebral artery one is between 2.4 and $4.6 \mathrm{~mm}$. On the other hand, the vertebral artery has $0.92-4.09 \mathrm{~mm}$ diameter, the posterior cerebral and the lower posterior cerebelar arteries ones are between 0.65 and $1.78 \mathrm{~mm}$.

Therefore, the human placenta is an excellent material for microsurgical technique training ${ }^{3,5,6}$.

\section{METHOD}

This study was developed at the Microsurgery Laboratory of the Hospital Beneficência Portuguesa de São Paulo and was also approved by the local ethics committee.

Twenty-five human placentas were analyzed to check if they were suitable for microvascular exercising. The available length and diameter of the vessel were measured and different microvascular anastomoses were performed. The used microsurgical materials were ${ }^{3,5,6}$ : forceps; microsurgery scissors; microsurgical knife; microsurgery needle holders; vascular clip appliers; neurovascular surgery clips; suture thread 10.0; Insulin syringe and needle; saline solution $0.9 \%$.

\section{RESULTS}

\section{Vascular suture (Fig 1)}

1. The placenta was submitted to hygienic cleaning and placed on the operative table for training.

2. The placenta was carefully examined under the microscope so that the best vessel could be chosen.

3. The superficial membrane was removed with the forceps.

4. The vascular segment was dissected and released from the placental estroma with microforceps, microscissors and microsurgical knife.

5. A latex piece was placed between the vessel and the placental estroma to facilitate the visualization under the microscope.

6. Two clips were applied to isolate around $1.5 \mathrm{~cm}$ of the vessel.

7. The adventitia recovering the artery wall was released with microforceps and after its dissection the tissue excess was cut with microscissors.

8. A superficial longitudinal linear incision was carefully made with the microsurgical knife in the isolated segment of the vessel.

9. The vessel was cleaned with physiological saline solution and small pieces of cotton. The vessel walls were identified.

10. The vascular wall was rebuilt with two microforceps and a suture thread 10.0.

11. One of these vessel walls was held with microforceps to support it for the suture.

12. The contra-lateral wall was held by its adventitious membrane, finalizing the transfixion of the vascular wall (be careful with excessive traction of the suture).

13. The whole work must be done under the microscope. Each suture must be tied three times. The clips must be removed after the end of every vascular synthesis and the vascular patency must be tested with the blood cross along the artery.

\section{TÉCNICAS MICROCIRÚRGICAS USANDO PLACENTA HUMANA}

Instituto de Ciências Neurológicas (ICNE), São Paulo SP, Brazil. Hospital Beneficiência Portuguesa de São Paulo, São Paulo SP, Brazil. Universidade Estadual de Campinas, Campinas SP, Brazil (UNICAMP): ${ }^{1}$ Neurosurgeon fellow of ICNE; ${ }^{2}$ Neurosurgeon of ICNE, Hospital Beneficência Portuguesa de São Paulo and UNICAMP; ${ }^{3}$ Director of ICNE, Chief of the Neurosurgery Division at UNICAMP.

Received 19 June 2008, received in final form 29 September 2008. Accepted 8 October 2008.

Dr. Flávio Ramalho Romero - Praça Amadeu Amaral 27 / 5 o andar-01327-010 São Paulo SP-Brasil. E-mail: romerocnr@gmail.com / icne@icne.com.br 

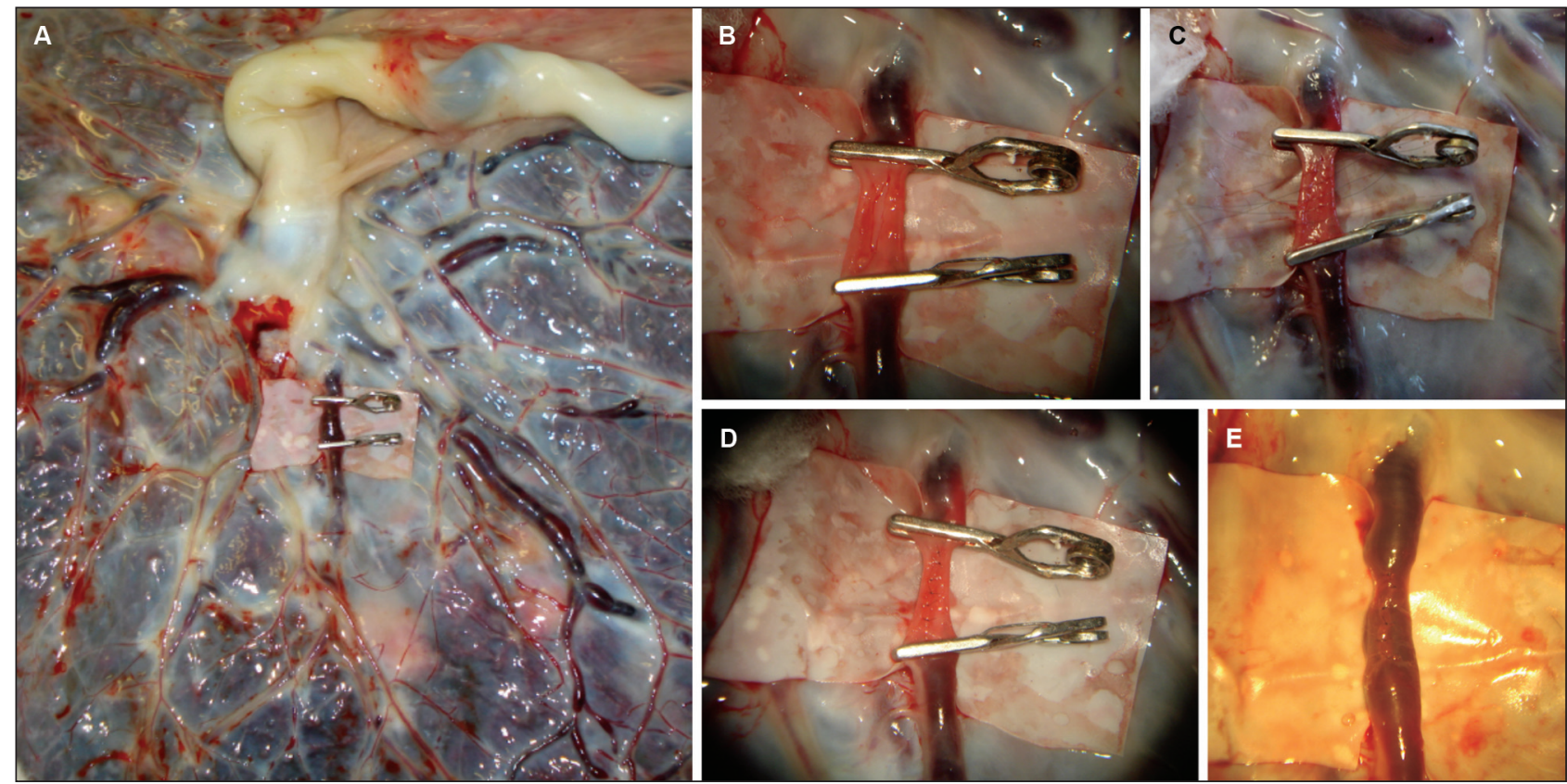

Fig 1. Microsurgical techniques to vascular suture in human placenta. (A) Choice of one artery, dissection, isolation and vascular occlusion using clips. (B) Opening vascular wall. (C) After passing the isolated suture. (D) Ending the suture. (E) Check the vascular patency after removing the vascular clips.
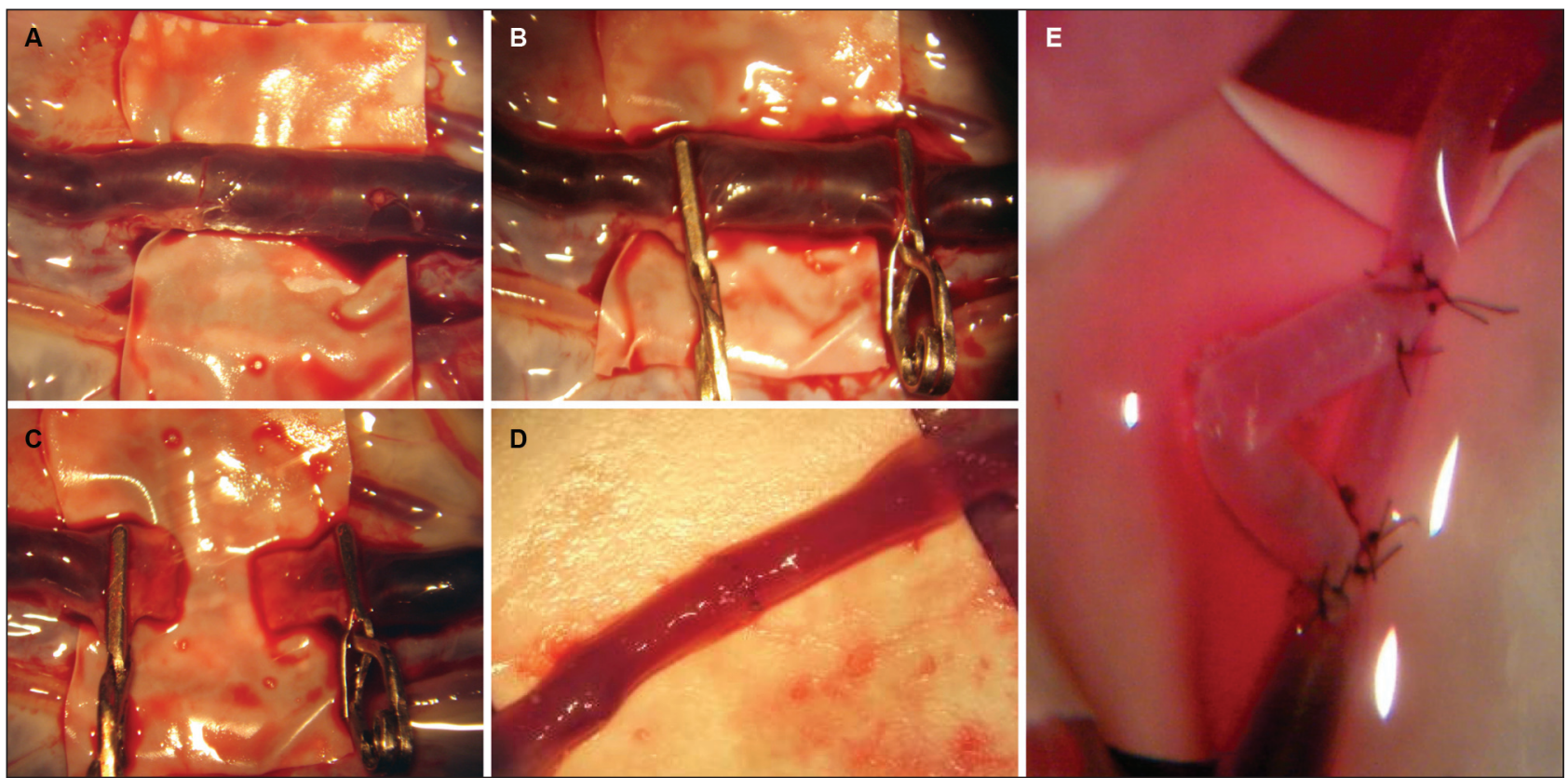

Fig 2. Example of ending-terminal anastomosis. (A) One vessel was choose, dissected and isolated. (B) Vascular chips were inserted and vascular flow was interrupted. (C) The vessel was transected. (D) After vascular suture and check of vascular patency. (E) Example of ending-lateral anatomosis - bypass. 


\section{Ending-terminal (Fig 2)}

1. Steps 1 to 7 were repeated.

2. One perpendicular cut was made in the arterial wall with a microscissor.

3. The vascular ending-terminal anastomosis was started with two microforceps and a suture thread 10.0.

4. The first suture was performed in the upper border of the vessel.

5. The second suture was performed in the lower border of the vessel.

6. The vessel was turned around with the microforceps to expose the posterior segment of the anastomosis.

7. The synthesis of this segment was performed by following the vascular suture steps 10 to 13. Only the adventitious layer must be manipulated.

8. The vessel was turned around to be worked in the anterior segment of the anastomosis.

9. The synthesis was concluded by following the vascular suture steps 10 to 13 in the anterior vessel segment.

\section{Ending-lateral (Fig 2)}

1. Steps 1 to 7 were repeated.

2. The artery that will serve as 'by-pass' was prepared according to the vascular suture steps 1 to 7 techniques previously described.

3. One oblique cut was made with a firm movement in the distal portion of the vessel to open an area in the terminal portion of the anastomosis.

4. In another vessel, the lateral portion was opened to create an elliptical hole.

5. The initial sutures were performed in the upper and lower portions of the elliptical hole.
6. First, the suture was performed in the posterior anastomotic segment.

7. This procedure was repeated in the anterior side.

8. The vascular patency was tested.

\section{DISCUSSION}

Although many neurosurgical training techniques are being used today such as mannequin head, virtual reality environment, animal or cadaver dissections, the human placenta can be easily and cheaply used ${ }^{1,2,3}$. As the placenta fetal surface is very similar to the cerebral arachnoid and the placenta vessels have the same size as the intracranial arteries, the dissection of human placenta is a very good microsurgical training.

In conclusion, the development of microsurgical techniques in laboratories is an important tool in training neurosurgeons and neurosurgeon fellows. We encourage the use of placenta in microsurgical training for neurosurgeons.

\section{REFERENCES}

1. Takeuchi M, Hayashi N, Hamada H, Matsumura N, Nishijo H, Endo S. A new training method to improve deep microsurgical skills using a mannequin head. Microsurgery 2008;28:168-170.

2. Kockro RA, Stadie A, Schwandt E, et al. A collaborative virtual reality environment for neurosurgical planning and training. Neurosurgery 2007;61(Suppl 2):S379-S391.

3. Ayoubi S, Ward P, Naik S, Sankaran M, et al. The use of placenta in a microvascular exercise. Neurosurgery 1992;30:252-254.

4. Panigel M. The human placenta: anatomy and morphology. Clin Obstet Gynaecol 1986;13:421-445.

5. Rhoton $\mathrm{Al} \mathrm{Jr}$. Cranial anatomy and surgical approaches, 2002

6. Salcman M. Current techniques in neurosurgery. 2.Ed. Philadelphia: Current Medicine, 1996:79-89. 